On oscillations and flutterings - a reply to Hamm and Fordyce

Niklas Janz ${ }^{* 1}$, Mariana P Braga ${ }^{1}$, Niklas Wahlberg ${ }^{2,3}$ and Sören Nylin ${ }^{1}$

* Corresponding author

${ }^{1}$ Department of Zoology, Stockholm University, 10691 Stockholm, Sweden

2 Laboratory of Genetics, Department of Biology, University of Turku, 20014 Turku, Finland

${ }^{3}$ Department of Biology, Lund University, 22362 Lund, Sweden

All data used in the manuscript are located in the Supplementary information and in DataDryad (doi:10.5061/dryad.p5v8g) 
In a recent paper in Evolution, Hamm and Fordyce (2015) published an analysis of "Patterns of host plant utilization and diversification in the brush-footed butterflies", which was presented as a test of our "oscillation hypothesis" (OH)(Janz et al. 2006; Janz and Nylin 2008; Nylin and Janz 2009). This study joins another recent paper (Hardy and Otto 2014) in claiming that macroevolutionary patterns among Nymphalid butterflies are not compatible with the $\mathrm{OH}$. While we were pleased to note their shared interest in macroevolutionary dynamics, we are concerned by their portrayal of the $\mathrm{OH}$ as well as the applicability and interpretation of their analyses. It should be noted that our comment deals only with the aspects of these papers that pertain to our hypothesis.

We focus on three of our primary concerns. The first is that one of the main predictions in the Hamm and Fordyce paper attributed to our $\mathrm{OH}$ is, in fact, not a prediction of the $\mathrm{OH}$. The second is that some of the models used were questionable choices for testing the hypothesis. The third concern is that the results of Hamm and Fordyce were ambiguous, and were actually often not in disagreement with the OH. Yet this ambiguity and potential agreement with the $\mathrm{OH}$ was not reflected in the authors' conclusions.

We suspect that some of the issues we have with the portrayal of the $\mathrm{OH}$ by Hamm \& Fordyce (and Hardy \& Otto) are caused by misunderstandings of our hypothesis, but some issues are probably caused by diverging views of how host shifts happen and how changes in host use may influence patterns of speciation. We will therefore begin with a short description of the $\mathrm{OH}$ to make it clear how we envision the process and where we think it differs from the alternatives expressed in these two papers. Next, we offer a reanalysis of the Hamm \& Fordyce dataset to demonstrate the troubling sensitivity of the models used to small changes in input variables and initial parameters. Finally, we argue that the conclusions regarding the $\mathrm{OH}$ made by Hamm \& Fordyce, as well as by Hardy \& Otto, are not substantiated. 


\section{THE OSCILLATION HYPOTHESIS}

Our first clarification is that the $\mathrm{OH}$ consists of two parts. The first is largely microevolutionary, and deals with how novel host plants are incorporated during host expansions and how as a consequence insect lineages can diversify in resource use. The second part is largely macroevolutionary, and predicts that these episodes of wider host use should lead to elevated diversification rates. These parts contribute different pieces to the puzzle that is the oscillation hypothesis. To some extent they are independent, in that evidence for one is not necessarily evidence for the other, but they are connected. The particulars of the microevolutionary part affects the specific patterns expected at the macroevolutionary level.

An essential tenet of the first part is that host shifts represent the endpoints of a process that involves at least two components: a host expansion followed by a loss of the ancestral host (specialization). This is, in a very condensed form, an example of a host range oscillation. The period of multiple host use may be brief, but can also extend over considerable lengths of time, and involve many host species. The main objective of this part of the hypothesis is to explain how novel plants are incorporated, but also how this affects the overall patterns of host plant colonization in a clade.

An early inspiration for the hypothesis came from the surprisingly high amount of host plant colonizations seen in the tribe Nymphalini (Janz et al. 2001), something that seemed to contradict observations of high host plant conservatism in butterflies (Ehrlich and Raven 1964; Janz and Nylin 1998). This observation has later been generalized as the "parasite paradox" (Agosta et al. 2010): groups of highly specialized parasites appear to shift hosts more readily than expected, given their conservative and specialized host associations. 
An important key in the resolution of this paradox is that there are two types of oscillations, and the difference between them is important (Fig. 1). The first kind involves colonizations of truly novel hosts that are not represented in the evolutionary past of the lineage in question. These should be rare events. The other kind represents recolonizations of ancestral hosts that have been lost and should be much more common (c.f. Ouvrard et al. 2015).

Any successful colonization requires some pre-existing ability to use the host. This may also appear paradoxical, but since selection can only act on expressed traits, the colonizing insect must show some positive realized fitness on the novel host already at the first encounter. This is known as ecological fitting (Janzen 1985; Agosta 2006), and is a necessary first step that sets the stage for further evolutionary modification of the trait. In the terminology of West-Eberhard, phenotypic plasticity in the insect can allow for phenotypic accommodation to a novel plant, later followed by genetic accommodation (West-Eberhard 2003; Nylin and Janz 2009).

In the case of recolonizations, it easy to see how there can be a significant overlap between the abilities required to feed on current hosts and those required to recolonize ancestral hosts that may have been lost through specialization. Indeed, it appears that the ability to use "lost" hosts may remain for a considerable time, making recolonizations common (Futuyma et al. 1995; Janz et al. 2001; Nylin et al. 2015). The result is a dynamic and complex pattern of host use, where species vary both in host range and actual hosts used, but appear to draw their hosts from a common pool of plants (Fig. 1). These changes in host use can be very rapid, as they are effectively changes in actual host use within a relatively stable potential host range. Colonization of such ancestral "lost" hosts is a form of recurrence homoplasy (West-Eberhard 2003; Nylin and Janz 2009), and we believe that it is crucial to understanding host range dynamics. 
Preexisting abilities can also be coopted to handle truly novel hosts through ecological fitting (Agosta and Klemens 2008; Agosta et al. 2010). Indeed, there is a sense in which "diversity begets diversity" also for host use (Janz et al. 2006); the potential overlap in the abilities required to handle existing and novel hosts should be higher in generalist species, due to their higher degree of plasticity in host preference and metabolization (García-Robledo and Horvitz 2011). The circumstances that may trigger such expansions are likely to be connected to climatic or other large-scale changes causing biotic turnover in ecological communities (Winkler et al. 2009; Jahner et al. 2011; Nyman et al. 2012; Hoberg and Brooks 2015).

Another important consequence is that it is important to maintain the identity of the hosts used when testing the $\mathrm{OH}$. This is the first point that Hamm and Fordyce failed to take into account. Implicit in their reasoning is a view where species can shift associations freely and instantaneously, unconstrained by history and plant phylogeny. This assumption is even made explicit in the other recent study claiming to find phylogenetic evidence against the $\mathrm{OH}$ (Hardy and Otto 2014), which used a similar methodology as Hamm and Fordyce.

Hardy and Otto (2014) rejected the $\mathrm{OH}$ and instead suggested an alternative scenario (the "musical chairs hypothesis") that implied instant shifts in host use among specialized species. To the extent that we can detect an alternative hypothesis in the Hamm and Fordyce paper, it appears to be based on similar thinking. It is worth noting however that Hardy and Otto had to incorporate a host use "lability" parameter in order to support their alternative hypothesis. Interestingly though, since the only states in the model were "specialist" and "generalist", the term represented lability in host range. In other words: host range oscillations. To the extent that their methodology is reliable (see below), their proposed solution relies on a "lability" term that is left unexplained. We 
believe that the first part of the $\mathrm{OH}$ outlined above provides an explanation for why some lineages have a "labile" host use and others don't (Fig. 1).

The second part of our hypothesis predicts that clades that have undergone the oscillations outlined above should be more species-rich compared to clades that have remained specialized on the same host (Janz et al. 2006). The argument behind this is that host expansions are necessary to provide the "fuel" for host-driven diversification, whatever the mechanism behind the actual speciation events. One possibility is that large geographic distributions tend to be associated with expanded host ranges (Päivinen et al. 2005; Jahner et al. 2011; Slove and Janz 2011; Slatyer et al. 2013), and that species with larger distributions will tend to become fragmented with time due to both neutral and adaptive processes (DeChaine and Martin 2004; Thompson 2005). Another possibility is sympatric speciation via "host races" specializing on different parts of the extended host repertoire. We do not expect the differences in speciation rates to be necessarily dramatic, but that they can nevertheless result in considerable amounts of diversification over time. It is worth pointing out that even if host plants can play a direct role in speciation (through for example host races), at least in the case of butterflies we expect the majority of these speciation events to be allopatric (Janz and Nylin 2008). In these cases, host associations only play a passive role in speciation, by increasing the likelihood of placing populations in vicariance. Most of the subsequent specialization would then occur in allopatry (c.f. Rich et al. 2008). Importantly, both types of host-driven speciation involve an element of specialization, either as a direct cause of speciation (as with host races) or as a passive consequence of speciation (as in allopatric speciation). Hence, in both cases, the process would soon run out of fuel unless it is somehow being "refueled" with new host associations. We argue that the oscillations described above can provide that fuel. 
We should thus expect a connection between host breadth and speciation, but this is not to say that generalist lineages should diversify at higher rates than specialist lineages. This may sound like a contradiction, but if polyphagy is evolutionarily ephemeral, any direct link between the two will be lost in time (Fig. 1). It is the repeated oscillations that we believe should lead to higher diversification rates, not the generalist state. Or, to put it another way, we do expect lineages with a wider range of potential hosts (as indicated by a more diverse host use in the clade) to diversify at a higher rate, but such lineages will often be made up of species with narrow ranges of actual hosts.

\section{TESTING THE RIGHT HYPOTHESIS}

In summary, host range oscillations should lead to diversification of host use, as well as increased "lability" in host range, and such repeated oscillations should increase the average rate of speciation. We want to emphasize though that we do not believe that such oscillations are the sole source of insect diversification. Precisely for this reason, we included a reciprocal sister group comparison in our first test of the hypothesis, as a means to test for causality behind the contrasts in species richness (Janz et al. 2006). We found that groups with higher host diversity were consistently more species-rich than their sister groups. On the other hand, the reciprocal test showed that groups with high species richness did not necessarily have higher host diversity than their sister clades. In other words, while higher host diversity was almost always followed by higher speciesrichness, some groups diversified for other reasons. Importantly though, in these cases the higher species richness should not be, and were not, accompanied by higher host diversity.

Hence, when Hamm and Fordyce in the first test in their article concluded that major differences in diversification rates among nymphalid butterflies were not correlated with wide host ranges, it was not the $\mathrm{OH}$ but our alternative hypothesis they tested. 
Their results were not surprising; we came to the same conclusion, albeit using a different methodology, nearly a decade ago (Janz et al. 2006).

Before going into details about the methodology, we would like to end this part by listing several explicit predictions that follow from the $\mathrm{OH}$, many of which should be testable (or have been tested) using phylogenetic methods (see references for more elaboration):

1. Clades that undergo oscillations should have higher diversification rates, so that clades with more diverse host use should tend to have more species than their sister clades. However, since species may diversify for many reasons, species rich clades should not necessarily be expected to have a more diverse host use (Janz et al. 2006).

2. While clades with diverse host use could be comprised of species specialized on different plants, such clades should also contain more generalist species (Nylin and Wahlberg 2008).

3. Following a host expansion, different descendant clades may follow different paths with regard to host use, but we should expect plants used in related clades to occasionally turn up as hosts within the more dominating plant theme, i.e. the broader collection of hosts used by this lineage of herbivores (Nylin and Wahlberg 2008).

4. Host shifts should predominantly involve ancestrally used host plants, forming part of a "potential" repertoire, i.e. we expect much recurrence homoplasy (Janz et al. 2001; Nylin and Janz 2009; Nylin et al. 2015).

5. Phylogenetic signal should be stronger for "potential" hosts than for actually used hosts (c.f. Janz et al. 2001; Nylin et al. 2015).

6. Truly novel host colonizations should be more common in generalists (Janz et al. 2001). 
7. Generalists should have larger geographic ranges, which would increase the likelihood of allopatric speciation (Slove and Janz 2011).

\section{CHOOSING THE RIGHT MODEL}

The second troubling aspect of the Hamm and Fordyce paper from our perspective is that some of the methods were not particularly well suited to test the $\mathrm{OH}$. Moreover, the results were ambiguous; some outcomes were not in agreement with the $\mathrm{OH}$, but many were. For reasons that are not clear to us, the authors chose to emphasize the deviating results. Here, we focus on one case that is particularly illustrative, for several reasons. Much of this critique also applies to the analyses by Hardy and Otto (2014).

Hamm \& Fordyce estimated diversification rates in relation to host plant breadth across the phylogeny, using the models BiSSE (with host range coded as a binary character) and MuSSE (with host range coded as a multistate character), as implemented in the $\mathrm{R}$ package "diversitree" (FitzJohn 2012). As should be clear from the preceding section, an obvious shortcoming of these analyses is that they do not take into account which plants make up the host range. As described above, the history of host use is an important predictor for future host colonizations in the OH (Janz et al. 2001; Nylin et al. 2015). Moreover, there was no measure of host plant relatedness, only a uniform scale of number of plant families used. Again, this could also have important consequences for the outcome.

Nevertheless, even setting these caveats aside, the analyses gave very different results depending on how host range was coded. When using a binary coding, and restricting the "specialist" state to genera using only one family, the model yielded a result where specialists had higher rates of diversification than generalists. This result could be construed as going against the $\mathrm{OH}$ (with the important caveats given above regarding other sources of diversification, as well as actual vs. potential hosts). However, with all 
other methods of coding, the results were in agreement even with a simplified interpretation of the $\mathrm{OH}$ that states that generalists should have higher diversification rates than specialists. The authors argued that more inclusive definitions of specialists (such as being restricted to two plant families or one order) are not "biologically informative", a statement that we find quite remarkable, and rather unsubstantiated. What is interesting is that different ways of coding host range yielded such different results, and it would have been interesting to see a more thorough discussion of this. On the face of it, it does seem a little counterintuitive that speciation events are shifted towards the generalist category when this category is made less inclusive. This in itself should be a reason for some concern.

Interestingly, the MuSSE results indicated that both mono- and polyphagous states had higher diversification rates than intermediate states. This also appears somewhat puzzling, but may perhaps be explained by the large subfamily of Satyrinae - many of which are specialized on grasses - which presumably accounts for much of the diversification on specialist branches. The diversification of Satyrinae on the geographically widespread grasses is an interesting special case, which we discussed in our original publication and that has also seen more extensive treatment since then (Peña and Wahlberg 2008; Peña et al. 2010).

As tests of the $\mathrm{OH}$, another significant limitation of the BiSSE and MuSSE models is that they only allow for anagenetic changes in host range. That is, specialization and colonization cannot happen during speciation events, something that is highly problematic when the hypothesis under test predicts a relationship between speciation and changes in host range. In this respect, the Hardy \& Otto analysis did somewhat better, as they also used the BiSSE-ness alternative, which does allow for cladogenetic change in host use. 


\section{A RE-ANALYSIS}

To further explore the usefulness of the "SSE" modeling approach, we have reanalyzed the dataset (available at http://doi.org/10.5061/dryad.p5v8g) using the similar model ClaSSE (Cladogenetic State change Speciation and Extinction), also available in the diversitree package. Like BiSSE-ness, ClaSSE can explicitly model both anagenetic and cladogenetic state changes (Goldberg and Igić 2012), making it a somewhat more realistic choice over BiSSE and MuSSE. See Supplementary Material 1 for a description of the method and a more detailed account of the results.

First, according to the estimated posterior probability distribution of each parameter, our results correspond qualitatively with the results from Hamm and Fordyce, for the parameters that represent anagenetic change (transition rates). Note, however, that the inclusion of cladogenetic state change alters the results related to speciation rates (Fig. S1). Even when the "specialist" state was restricted to genera using only one family, diversification rates were higher for generalists whether it was linked to state change or not. The highest rate was the speciation of generalists without state change, followed by the speciation of generalist lineages into specialist daughter species. Speciation rates of parent specialist lineages with and without state change were similar and at least 10 times smaller than that of generalist lineages.

In order to understand why our results differed from those of Hamm and Fordyce, we evaluated the sensitivity of the analysis to the initial parameters of the maximum likelihood estimation. Surprisingly, small changes in such parameters yielded very different results (Fig S2), showing that the "SSE" modeling approach lacks robustness.

Evidently, relatively small changes to the model were enough to produce results that are in accordance with the expectations from a simplified version of the $\mathrm{OH}$, even if there was still a high sensitivity to character coding and model specification. Moreover, the 
ClaSSE model shares many of the other issues with regard to testing the $\mathrm{OH}$, in that it ignores all of the qualifications given above. Finally, and perhaps even more disturbing, others have cautioned against current implementations of these methods for investigating trait-dependent diversification at all, as they tend to produce spurious associations between character state and speciation rate (Type I error) due to phylogenetic pseudoreplication and other unknown model inadequacies (Maddison and FitzJohn 2015; Rabosky and Goldberg 2015).

\section{CONCLUSIONS}

The main conclusion from our reanalysis of Hamm \& Fordyce's data is that the "SSE" models are so sensitive to small changes in input variables and model specification that they can potentially give any result you want. In the light of this, and the other problems mentioned above, we would caution against using the "SSE" models to test the $\mathrm{OH}$ specifically and for testing trait-based diversification in general.

We do welcome the interest in the oscillation hypothesis, and look forward to a continued discussion of it in the future, for example on how to work out accurate and fair tests of it. To do this, we consider it crucial to acknowledge the processes that we mean are causing the host range oscillations, and to preserve the identity of the plants in the insects' host ranges. This may also serve to resolve some of the apparent conflicts between our hypothesis and the alternatives recently presented by Hamm \& Fordyce and Hardy \& Otto. For example, the evolutionary "tinkering" among descendant species, where they mix and match between plants contained within an ancestral oscillation, may provide an explanation for the host use "lability" suggested by Hardy and Otto (2014).

Oh, and the word "oscillation" should not be taken too literally. We do not mean to imply that host expansions happen with regular frequency and amplitude. Perhaps "fluttering", 
as suggested by Hamm and Fordyce, would be a more accurate description. But then again, wouldn't "the fluttering hypothesis" sound kind of silly?

\section{LITERATURE CITED}

Agosta, S.J., 2006. On ecological fitting, plant-insect associations, herbivore host shifts, and host plant selection. Oikos. 114(3):556-565.

Agosta, S.J. \& J.A. Klemens. 2008. Ecological fitting by phenotypically flexible genotypes: implications for species associations, community assembly and evolution. Ecol. Lett. 11(11):1123-1134.

Agosta, S.J., N. Janz \& D.R. Brooks. 2010. How specialists can be generalists: resolving the "parasite paradox" and implications for emerging infectious disease. Zoologia. 27(2):151-162.

DeChaine, E.G. \& A.P. Martin. 2004. Historic cycles of fragmentation and expansion in Parnassius smintheus (Papilionidae) inferred using mitochondrial DNA. Evolution. 58(1):113-127.

Ehrlich, P.R. \& P.H. Raven. 1964. Butterflies and plants: a study in coevolution. Evolution. 18:586-608.

FitzJohn, R.G., 2012. Diversitree : comparative phylogenetic analyses of diversification in R. Methods. Ecol. Evol. 3(6):1084-1092.

Futuyma, D.J., M.C. Keese \& D.J. Funk. 1995. Genetic constraints on macroevolution: the evolution of host affiliation in the leaf beetle genus Ophraella. Evolution. 49:797-809. García-Robledo, C. \& C.C. Horvitz. 2011. Jack of all trades masters novel host plants: positive genetic correlations in specialist and generalist insect herbivores expanding their diets to novel hosts. Journal. of. Evolutionary. Biology. 25(1):38-53. 
Goldberg, E.E. \& B. Igić. 2012. Tempo and mode in plant breeding system evolution. Evolution. 66(12):3701-3709.

Hamm, C.A. \& J.A. Fordyce. 2015. Patterns of host plant utilization and diversification in the brush-footed butterflies. Evolution. 69(3):589-601.

Hardy, N.B. \& S.P. Otto. 2014. Specialization and generalization in the diversification of phytophagous insects: tests of the musical chairs and oscillation hypotheses. Proc. R. Soc. B. 281(1795):20132960.

Hoberg, E.P. \& D.R. Brooks. 2015. Evolution in action: climate change, biodiversity dynamics and emerging infectious disease. Philosophical. Transactions. of. the. Royal. Society. of. London. B:. Biological. Sciences. 370(1665):20130553.

Jahner, J.P., M.M. Bonilla, K.J. Badik, A.M. Shapiro \& M.L. Forister. 2011. Use of exotic hosts by lepidoptera: widespread species colonize more novel hosts. Evolution. 65(9):2719-2724.

Janz, N. \& Nylin, S. 2008, The oscillation hypothesis of host plant-range and speciation, in Tilmon, K. J. ed. Specialization, Speciation, and Radiation: the Evolutionary Biology of Herbivorous Insects. University of California Press, Berkeley, California:203-15.

Janz, N. \& S. Nylin. 1998. Butterflies and plants: a phylogenetic study. Evolution. 52:486502.

Janz, N., S. Nylin \& K. Nyblom. 2001. Evolutionary dynamics of host plant specialization: a case study of the tribe Nymphalini. Evolution. 55:783-796.

Janz, N., S. Nylin \& N. Wahlberg. 2006. Diversity begets diversity: host expansions and the diversification of plant-feeding insects. BMC. Evolutionary. Biolology. 6:4.

Janzen, D.H., 1985. On ecological fitting. Oikos. 45(3):308-310. 
Maddison, W.P. \& R.G. FitzJohn. 2015. The unsolved challenge to phylogenetic correlation tests for categorical characters. Syst. Biol. 64(1):127-136.

Nylin, S. \& N. Janz. 2009. Butterfly host plant range: an example of plasticity as a promoter of speciation? Evol. Ecol. 23(1):137-146.

Nylin, S. \& N. Wahlberg. 2008. Does plasticity drive speciation? Host-plant shifts and diversification in nymphaline butterflies (Lepidoptera : Nymphalidae) during the tertiary. Biol. J. Linn. Soc. 94(1):115-130.

Nylin, S., L. Söderlind, G. Gamberale-Stille, H. Audusseau, M.d.l.P. Celorio Mancera, N. Janz \& F.A.H. Sperling. 2015. Vestiges of an ancestral host plant: preference and performance in the butterfly Polygonia faunus and its sister species P. c-album. Ecol. Entomol. 40(3):307-315.

Nyman, T., H.P. Linder, C. Peña, T. Malm \& N. Wahlberg. 2012. Climate-driven diversity dynamics in plants and plant-feeding insects. Ecol. Lett.:no-no.

Ouvrard, D., P. Chalise \& D.M. Percy. 2015. Host-plant leaps versus host-plant shuffle: a global survey reveals contrasting patterns in an oligophagous insect group (Hemiptera, Psylloidea). Systematics. and. Biodiversity..

Päivinen, J., A. Grapputo, V. Kaitala, A. Komonen, J.S. Kotiaho, K. Saarinen \& N. Wahlberg. 2005. Negative density-distribution relationship in butterflies. BMC. Biol. 3(1):5.

Peña, C. \& N. Wahlberg. 2008. Prehistorical climate change increased diversification of a group of butterflies. Biol. Lett. 4(3):274-278.

Peña, C., S. Nylin \& N. Wahlberg. 2010. The radiation of Satyrini butterflies (Nymphalidae: Satyrinae): a challenge for phylogenetic methods. Zool. J. Linn. Soc. 161:64-87. 
Rabosky, D.L. \& E.E. Goldberg. 2015. Model inadequacy and mistaken inferences of traitdependent speciation. Syst. Biol. 64(2):340-355.

Rich, K.A., J.N. Thompson \& C.C. Fernandez. 2008. Diverse historical processes shape deep phylogeographical divergence in the pollinating seed parasite Greya politella. Mol. Ecol. 17(10):2430-2448.

Slatyer, R.A., M. Hirst, J.P. Sexton \& D. Kleijn. 2013. Niche breadth predicts geographical range size: a general ecological pattern. Ecol. Lett. 16(8):1104-1114.

Slove, J. \& N. Janz. 2011. The relationship between diet breadth and geographic range size in the butterfly subfamily Nymphalinae - a study of global scale. PLoS. One. 6(1):e16057.

Thompson, J.N.2005. The geographic mosaic of coevolution. University Of Chicago Press, Chicago.

West-Eberhard, M.J.2003. Developmental plasticity and evolution. Oxford University Press, New York.

Winkler, I.S., C. Mitter \& S.J. Scheffer. 2009. Repeated climate-linked host shifts have promoted diversification in a temperate clade of leaf-mining flies. P. Natl. Acad. Sci. USA. 106(43):18103-18108. 


\section{FIGURE LEGENDS}

Fig. 1. A schematic representation of oscillations in actual and potential host range. The ancestral state (species 1-2) is specialization on host A. The arrow indicates an event where the host range was expanded to also include the novel hosts B and C. Species 3-6 later specialized on one of A-C as actual hosts, but retained some capacity to potentially use all of the hosts (a-c). Thus, species 7 could later again expand its actual host range to include A-C. Note that there are more species in the clade consisting of species 3-7, with more diverse host use than in the sister clade (species 2), but that actual polyphagy (species 7) is recent and has not promoted diversification.

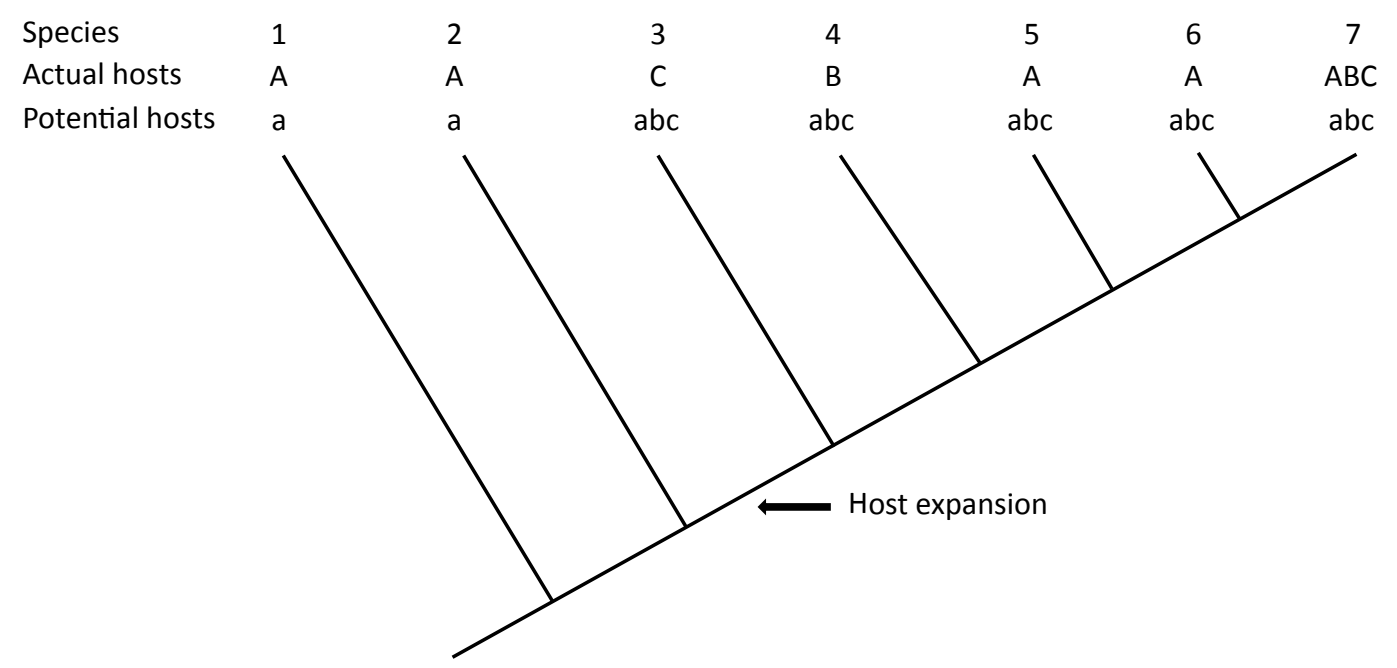

